\title{
Über Sulfide des Rubidiums und Cäsiums.
}

\author{
Von
}

\author{
Wilhelm Biltz und Ernst Wilke-Dörfurt.
}

Mit 2 Figuren im Text.

Die Gründe, welche uns vor einiger Zeit veranlalst hatten, das Studium der Rubidium- und Cäsiumsulfide und -Polysulfide aufzunehmen, waren die folgenden: Erstens war zu erwarten, dafs die genannten Netalle sich vor ihren Verwandten besonders gut zur Bereitung beständiger und kristallisationsfähiger Polysulfide eignen würden und zweitens war durch die Lehre von den heterogenen Gleichgewichten eine Möglichkeit an die Hand gegeben, ein passendes Ausgangsmaterial vorausgesetzt, auch unabhängig von den präparativen Versuchen zu entscheiden, welche der möglichen Sulfide existieren.

Die erste Vermutung ist, wie an anderer Stelle gezeigt wurde, ${ }^{1}$ durch die Eigenschaften der Rubidium- und Cäsiumpentasulfide vollkommen bestätigt und, wie ebenda dargelegt wurde, der Befund somit in Übereinstimmung mit einer theoretischen Vorstellung über die Beeinflussung der Beständigkeit gewisser negativer Komplexe durch die Natur des positiven Verbindungsbestandteiles erwiesen worden. Diese Vorstellung hatte sich aus den im nachstehenden Zitate enthaltenen Tatsachen entwickelt. „Wenn man die Polysulfidradikale als „homogene Komplexe“ den Polyhalogenradikalen der gleichen Beschaffenheit, ferner den aus mehreren Elementen oder Elementgruppen gebildeten Radikalen höher komplexer Verbindungen an die Seite stellt, so erscheint von vornherein ein Analogieschlufs aus der Beständigkeit dieser auf das Verhalten jener möglich. Wir begegnen nun der auffälligen Tatsache, dafs vielfach

1 Ber. deutsch. chem. Ges. 38 (1905), 123.

Z. anorg. Chem. Bd. 48 . 
da, wo es sich um den Aufbau negativer, komplexer Radikale handelt, die Verwendung der Alkalimetalle von höherem Atomgewicht von gröfstem Vorteil ist. Kaliumpentajodid ist nicht bekannt, Cäsiumpentajodid wurde als wohldefinierter Körper von WeLLs und seinen Mitarbeitern erhalten, wie denn überhaupt die Chemie der Polyhalogenverbindungen erst durch Verwendung der Rubidium- und Cäsiumsalze präparativ erschlossen worden ist. In der Reihe der Verbindungen, deren negative Komplexe aus Metall und Halogen bestehen, zeigt sich eine ähnliche Regel. Es konnten des weiteren auch Cäsium- und Rubidiumalaune mit Titansulfat erhalten werden." 1

In der nachstehenden Abhandlung sind zunächst unsere Erfahrungen über die Darstellung der niederen Sulfide und Polysulfide des Rubidiums und Cäsiums aus wässerigen Lösungen nebst den analytischen Belegen enthalten; in einem zweiten Teile ist der thermoanalytische Nachweis der Tri-, Tetra-, Penta- und Hexasulfide geführt.

a) Monosulfide und Hydrosulfide.

$\mathrm{Zu}$ den Monosulfiden und Hydrosulfiden des Rubidiums und Cäsiums zu gelangen, macht keine Schwierigkeiten, wenn die für die anderen Alkalimetalle bekannten Vorschriften von Benzeuros eingehalten und bei der Bereitung der Sulfidlösungen die in unserer früheren Mitteilung angegebenen Mafsregeln beachtet werden; wie dort genauer begründet ist, ${ }^{2}$ erfordert die Volumzunahme einer Alkalilauge beim Einleiten von Schwefelwasserstoff, sowie die Tatsache, dafs von einer solchen noch über das Hydrosulfid hinaus Schwefelwasserstoff absorbiert wird, eine jedesmalige vollständige Analyse und

1 Weiteres vergl. l. c. Anmerkung.

2 Unsere frühere Angabe, der Überschứs an physikalisch gelöstem Schwefelwasserstoff in gesättigten Kaliumhydrosulfidlösungen richte sich nach der Konzentration der Laugen, bedarf in dieser Fassung der Berichtigung. Vergleicht man diejenige Anzahl Gramme Schwefelwasserstoff, die in $1 \mathrm{ccm} \mathrm{7.9,}$ 3.14 und 0.21 fach normaler, gesättigter Lauge im Überschusse enthalten sind, nämlich jeweils $0.0024 \mathrm{~g}, 0.0035 \mathrm{~g}$ und $0.0030 \mathrm{~g}$, so sieht man, wie der Überschufs vielmehr nahezu der gleiche ist, aber - und hierdurch wird eine Beeinflussung der Schwefelwasserstofflöslichkeit durch die Anwesenheit des Alkalihydrosulfids bewiesen -, wie in jedem Falle eine kleinere Menge aufgenommen wird, als von reinem Wasser; nach Scroenfeld, Ann. 95 (1855), 1, löst $1 \mathrm{ccm}$ Wasser bei den hier in Frage kommenden Temperaturen, $2-8^{\circ}$, durchschnittlich $0.0057 \mathrm{~g}$. 
eine dieser entsprechende Korrektur, wenn durch Mischen der gawonnenen Sulfidlauge mit Hydroxydlösung eine stöchiometrisch zusammengesetzte Monosulfid-oder Hydrosulfidlösung erhalten werden soll.

Die Ausgangsmaterialien wurden, wie früher, zum Teil in sehr reinem Zustande käuflich bezogen. Einen Teil des Rubidiumhydroxyds bereiteten wir uns selbst nach dem Verfahren von H. ERDMANN $^{2}$ aus Rubidiumaluminiumalaun. Allerdings sind die Vorarbeiten hierzu: die Darstellung des zur fraktionierten Kristallisation nötigen Rubidiumeisenalauns, sowie dessen Aufarbeitung zu Hydroxyd zum mindesten im Mafsstabe des Laboratoriums verhältnismälsig recht zeitraubend. Die fraktionierte Kristallisation selbst wird indessen durch den hohen Temperaturkoeffizienten der Löslichkeit des Salzes sehr erleichtert. Als Mittel von je 2 Bestimmungen, bei denen von übersättigter Lösung einerseits, von reinem Wasser und festem Salz andererseits ausgegangen wurde, ergab sich die Löslichkeit des Rubidiumeisenalauns in Abhängigkeit von der Temperatur, wie folgt:

$\begin{array}{cc}\text { Temperatur } & \text { Löslichkeit in } \mathrm{g} \text { pro } 100 \mathrm{ccm} \text { Lösung } \\ 6.6^{\circ} & 4.55 \mathrm{~g} \\ 25 & 29.0 \\ 40 & 52.6\end{array}$

Für die Befreiung der Hydroxydlösungen von Karbonat, ihre Sättigung mit Schwefelwasserstoff und die Analysen gelten die in unserer zitierten Abhandlung gegebenen Vorschriften.

Rubidiummonosulfid, $\mathrm{Rb}_{2} \mathrm{~S}_{4} 4 \mathrm{H}_{2} \mathrm{O}$.

A. Eine Monosulfidlösung von richtiger Zusammensetzung wurde mit Alkohol gefällt, das erhaltene Öl im Überschusse des Fällungsmittels gelöst und die Lösung mit Ather versetzt. Wurde das Auflösen mit Alkohol und Fällen mit Äther mehrfach wiederholt, so schieden sich aus dem derart konzentrierten Öl nach 24 Stunden im Vakuum feine, weilse, äufserst zerfliefsliche Kristallmassen ab, die nach eintägigem Trocknen im Vakuumexsikkator auf Ton folgende Analysenzahlen lieferten:

$0.4022 \mathrm{~g}$ Substanz wurden in $50 \mathrm{ccm}$ Wasser gelöst; je $10 \mathrm{ccm}$ verbrauchten in 2 Bestimmungen je $5.88 \mathrm{ccm}{ }^{1} / 10 \mathrm{n} . \mathrm{AgNO}_{3}$; 10 weitere $\mathrm{ccm} 5.85 \mathrm{ccm} 1 / 10 \mathrm{n}$. HCl.

Ber. S 11.65; Rb 62.15. - Gef. S 11.7; Rb 62.2, Rb:S = $2: 1.05$.

${ }^{1}$ Arch. d. Pharm. 232 (1894), 1. 
Das Präparat war karbonat- und polysulfidfrei.

B. Beliefs man eine im Wasserstoffstrome sehr stark eingeengte Rubidiummonosulfidlösung im Vakuumexsikkator über Chlorcalcium, so erhielt man nach 2 Wochen aus der ein wenig gelb gewordenen Mutterlauge ebenfalls eine reichliche Kristallisation rein weifsen Monosulfids.

$1.1726 \mathrm{~g}$ wurden in $100 \mathrm{ccm}$ Wasser gelöst, je $10 \mathrm{ccm}$ dieser Lösung verbrauchten in 2 Bestimmungen je $8.49 \mathrm{~cm} 1 / 10 \mathrm{n} . \mathrm{AgNO}_{3}$, je $10 \mathrm{ccm}$ verbrauchten in 2 weiteren Bestimmungen je 8.55 und $8.57 \mathrm{ccm} 1 / 10 \mathrm{n} . \mathrm{HCl}$.

Ber. S 11.65; Rb 62.15. - Gef. S 11.6, Rb 62.3 und 62.5, $\mathrm{Rb}: \mathrm{S}=2: 0.992$.

\section{Rubidiumbydrosulfid RbSH.}

$5 \mathrm{ccm}$ einer 5.9 fach normalen Hydrosulfidlösung lieferten unter den erwähnten Bedingungen nach 6 Tagen eine Kristallisation von weifsglänzenden, undeutlich ausgebildeten, sehr zerfliefslichen Nadeln.

$0.4085 \mathrm{~g}$ Substanz (7 Tage im Vakuum getrocknet) wurden in $50 \mathrm{ccm}$ Wasser gelöst; $10 \mathrm{ccm}$ verbrauchten $13.66 \mathrm{bzw} .13 .65 \mathrm{ccm}$ $1 / 10$ n. $\mathrm{AgNO}_{3} ; 10 \mathrm{ccm}$ verbrauchten 6.76 bzw. $6.72 \mathrm{ccm} 1 / 10 \mathrm{n} . \mathrm{HCl}$.

Ber. S 27.04; Rb 72.11. - Gef. S 26.80, 26.78; Rb 70.7, $70.3 ; \mathrm{Rb}: \mathrm{S}=1: 1.02$.

\section{Cäsiummonosulfid $\mathrm{Cs}_{2} \mathrm{~S} .4 \mathrm{H}_{2} \mathrm{O}$.}

Von dem ganz wie Rubidiummonosulfid B dargestelìten Präparate verbrauchten $0.13236 \mathrm{~g} 6.90 \mathrm{~cm}{ }^{1} / 10 \mathrm{n} . \mathrm{AgNO}_{3}, 0.15770 \mathrm{~g}$ 8.81 bzw. $8.69 \mathrm{ccm} 1 / 10$ n. $\mathrm{AgNO}_{3}$ und $8.55 \mathrm{ccm} 1 / 10$ n. HCl.

Ber. S 8.67; Cs 71.85. - Gef. S 8.36, 8.95, 8.83; Cs 72.0 $\mathrm{Cs}: \mathrm{S}=2: 1.02$.

\section{Cäsiumhydrosulfid CsSH.}

Cäsiumhydrosulfid konnte ebenfalls als reinweilse hygroskopische Kristallmasse von erwarteter Zusammensetzung erhalten werden. Spuren verunreinigenden Polysulfids gaben sich beim Ansäuern der Lösung durch ganz geringe Schwefelabscheidung zu erkennen.

0.18248 g Substanz verbrauchten $21.32 \mathrm{ccm} 1 / 10 \mathrm{n} . \mathrm{AgNO}_{3}$, bzw. $11.10 \mathrm{ccm} 1 / 10$ n. HCl. Ber. S 19.32; Cs 80.07. Gef. S 18.73; Cs 80.8 , Cs $: S=1: 0.960$. 
Reine, wasserfreie Monosulfide von Rubidium und Cäsium lassen sich aus ihren Hydraten durch Entwässern ebensowenig gewinnen, wie die der übrigen Alkalimetalle. Im Schwefelwasserstoffstrome zerflofs ein Rubidiummonosulfid zunächst unter Gasabsorption; wurde sodann im Paraffinbade bis $200^{\circ}$ erhitzt, so hinterblieb eine gleichmälsig weifse Kristallmasse, die $13.9 \%$ weniger wog, als die angewandte Substanz. Für den Übergang in ein Dihydrat, $\mathrm{Rb}_{2} \mathrm{~S} .2 \mathrm{H}_{2} \mathrm{O}$ berechnet sich ein Gewichtsverlust von $13.1 \%$. Erhitzte man vorsichtig mit freier Fächelflamme, so entstanden augenblicklich in der weifsen Salzmasse rote Flecke. Durch längeres Erhitzen auf höhere Temperatur konnte indessen weder im Schwefelwasserstoff. noch im Wasserstoffstrome ein reines wasserfreies Präparat erhalten werden, weil im ersten Falle Polysulfidbildung, im zweiten Hydrolyse unvermeidbar war. Immerhin zeigt der augenblickliche Farbumschlag bei einer Temperatur, die weit unterhalb derjenigen merklicher Schwefelwasserstoffzersetzung liegt, im Verein mit den zu schildernden Versuchen über wasserfreie Disulfide, dals die Farbe der wasserfreien, niederen Sulfide von Rubidium und Cäsium rot, also die gleiche, wie bei derartigen Verbindungen der leichteren Alkalimetalle ist.

\section{b. Polysulfide.}

Durch Eintragen von Schwefel in Monosulfidlösungen und Einengen zur Kristallisation kann man nicht zu reinen Di- oder Trisulfiden, wohl aber zu Tetrasulfiden gelangen. Die folgenden Versuche zeigen, dafs in allen Fällen statt Disulfid und Trisulfid ein höheres Sulfid in einer natürlich geringeren Ausbeute entsteht. Die Neigung, Tetrasulfid zu bilden, ist besonders beim Cäsium scharf ausgeprägt. Es folgt somit aufs neue, dafs in Lösungen niedriger Polysulfide Tetrasulfide die beständigsten sind, also dasselbe Resultat, das KÜsTER ${ }^{1}$ durch Messungen der Hydrolyse und Potentialmessungen an Natriumpolysulfidlösungen mit voller Schärfe erzielt hat und das in der Kaliumreihe auch aus Versuchen von BLoxam ${ }^{2}$ ersichtlich wird, der, als die Darstellung von $\mathrm{K}_{2} \mathrm{~S}_{3}$ und $\mathrm{K}_{2} \mathrm{~S}_{2}$ aus Hydrosulfid und Schwefel beabsichtigt war, $\mathrm{K}_{2} \mathrm{~S}_{4}$ und $\mathrm{K}_{2} \mathrm{~S}_{3}$ erhielt.

Wurde in eine etwa 5 fach normale Rubidiummonosulfidlösung die für Disulfid berechnete Schwefelmenge eingetragen, so färbte

1 Z. anorg. Chem. 43 (1905), 53; 44 (1905), 431.

${ }^{2}$ J. Chem. Soe. 77 (1900), 752. 
sich die Lösung durch Schwefelaufnahme rotgelb. Nach dem Einengen im Wasserstoffstrome erhielt man nach 4-7 Tagen eine undeutlich kristallisierte, in dunkelroter Mutterlauge befindliche, lebergelbe Masse, die nach 2 Tagen im Vakuumexsikkator ziemlich gleichmälsig hellgelb wurde. Das Produkt löst sich in Wasser klar und intensiv gelb, in Salzsäure unter Schwefelabscheidung. Die mikroskopische Betrachtung und die Analyse ${ }^{1}$ erwiesen die Ungleichmärsigkeit des gewonnenen Produktes.

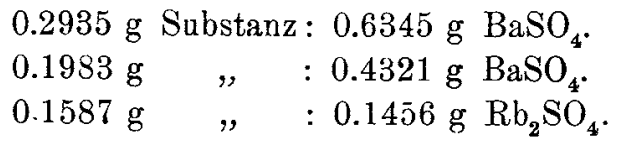

Ber. für $\mathrm{Rb}_{2} \mathrm{~S}_{2}$, $\mathrm{S} 27.27$; Rb 72.73. - Gef. $\mathrm{S} 29.69$, 29.92; $\mathrm{Rb}$ 58.75; $\mathrm{Rb}: \mathrm{S}=2: 2.71$.

Die Zusammensetzung liegt also zwischen Di- und Trisulfid. Eine bis zur Sirupsdicke eingeengte, entsprechend bereitete „Cäsiumdisulfid"-Lösung war ebenfalls rot und lieferte eine geringe Ausbeute $(0.4 \mathrm{~g})$ blättrig zusammengewachsener Nadeln, deren Farbe im feuchten Zustande rot, nach zweitägigem Trocknen rein hellgelb erschien. Die Zusammensetzung der Kristalle liegt zwischen Triund Tetrasulfid.

$$
\begin{array}{llllll}
0.0460 \mathrm{~g} \text { Substanz: } & 0.0431 \mathrm{~g} \mathrm{Cs}_{2} \mathrm{SO}_{4} \text { und } 0.0996 \mathrm{~g} \mathrm{BaSO}_{4} \text {. } \\
0.0480 \mathrm{~g} ": \quad & 0.0451 \mathrm{~g} \mathrm{Cs}_{2} \mathrm{SO}_{4}, \quad 0.1024 \mathrm{~g} \mathrm{BaSO}_{4} \text {. }
\end{array}
$$

Ber. für $\mathrm{Cs}_{2} \mathrm{~S}_{2}$, S 19.44; Cs 80.56. - Gef. S 29.7, 29.5; Cs $68.8,69.0 ;$ Cs : $\mathrm{S}=2: 3.56$.

1 Zur Analyse der Polysulfide führt man entweder die Substanz durch Oxydation mit ammoniakalischem Wasserstoffsuperoxyd in Sulfat über: etwa $0.2-0.3 \mathrm{~g}$ werden in einem Gemisch von $3 \mathrm{ccm}$ absolutem Alkohol und $7 \mathrm{ccm}$ konzentriertem Ammoniak gelöst und mit einer Mischung von $10 \mathrm{cem} 5 \%$ igem Wasserstoffsuperoxyd und $5 \mathrm{ccm}$ konzentriertem Ammoniak oxydiert; Alkohol, Ammoniak und überschüssiges Wasserstoffsuperoxyd werden durch Erhitzen auf dem Wasserbade entfernt. Der Alkaligehalt wird durch Abrösten einer zweiten Substanzprobe im Porzellantiegel, eventuell, wie dies bei Disulfiden bisweilen durchgeführt wurde, durch Titration mit 1/10 n. Säure bestimmt.

Viel bequemer, genauer und sparsamer ist es indessen, wenn raan die Probe trocken im Sauerstoffstrom röstet und die Röstgase in ammoniakalischem Wasserstoffsuperoxyd auffängt; die Wägung des entstandenen Sulfats ergibt den Alkaligehalt, die Fällung der vorgelegten Flüssigkeit mitsamt dem aufgelösten Röstprodukt mittels Baryumchlorid den Schwefel. 
Werden theoretisch zusammengesetzte ,Trisulfid"-Lösungen, die je nach der Konzentration rotgelb bis dunkelrot erscheinen, durch Einengen im Wasserstoffstrome oder durch langsames Eindunsten im Vakuumexsikkator zur Kristallisation gezwungen, so entsteht im Falle des Rubidiums eine je nach den Ausscheidungsbedingungen mehr oder minder gut ausgebildete gelbe bis rotgelbe, wasserhaltige Kristallisation eines Stoffes, in dem sich Rubidium zu Schwefel verhält wie $2: 3.13$.

a) Langsam entstandene Kristalle, hart, rotgelb, von sargförmigem Habitus:

$0.1024 \mathrm{~g}$ Substanz: $0.2596 \mathrm{~g} \mathrm{BaSO}_{4}$.

$0.1259 \mathrm{~g}, \quad: 0.3225 \mathrm{~g} \mathrm{BaSO}_{4}$.

$0.2268 \mathrm{~g} \quad: \quad: 0.2115 \mathrm{~g} \mathrm{Rb}_{2} \mathrm{SO}_{4}$.

Ber. für $\mathrm{Rb}_{2} \mathrm{~S}_{3}, \mathrm{~S} 36.00$; Rb 64.00. — Gef. S 34.81, 35.18; $\mathrm{Rb} 59.71$.

b) Undeutlich kristallisierte, lebergelbe Masse.

$0.1262 \mathrm{~g}$ Substanz: $0.3334 \mathrm{~g} \mathrm{BaSO}$.

$0.1614 \mathrm{~g}, \quad: 0.4204 \mathrm{~g} \mathrm{BaSO}_{4}$

$0.3250 \mathrm{~g} \quad, \quad: 0.3094 \mathrm{~g} \mathrm{Rb}_{2} \mathrm{SO}_{4}$.

Gef. S 36.28, 35.77; Rb. 60.96 .

Durch Auflösen der vereinigten Ausbeuten in Wasser und Fällen mit Alkohol gelangten wir ebensowenig zu reinem Trisulfid; die erhaltenen gelben grofsblättrigen Kristalle enthielten vielmehr Rubidium und Schwefel im Verhältnisse $2: 2.64$.

Im Falle des Cäsiums entstanden aus einer Lösung, die genau so, wie eine Pentasulfidlösung aussah, lange, harte, rotgelbe, gelb durchscheinende Nadeln, die im Exsikkator etwas verwitterten und im wesentlichen aus kristallwasserhaltigem Cäsiumtetrasulfid bestanden.

$0.1966 \mathrm{~g}$ Substanz: $0.4295 \mathrm{~g} \mathrm{BaSO}_{4}$.

$0.2144 \mathrm{~g} \quad, \quad: 0.4668 \mathrm{~g} \mathrm{BaSO}_{4}$.

$0.1996 \mathrm{~g} \quad, \quad: 0.1743 \mathrm{~g} \mathrm{Cs}_{2} \mathrm{SO}_{4}$.

Ber. für $\mathrm{Cs}_{2} \mathrm{~S}_{4} 11_{2} \mathrm{H}_{2} \mathrm{O}, \mathrm{S} 30.45 ; \mathrm{Cs} 63.14 ; \mathrm{H}_{2} \mathrm{O} 6.41$.

Gef. S 29.93, 29.90; Cs 64.14; $\mathrm{H}_{2} \mathrm{O}$ aus der Diff. 5.94; Cs:S $=2: 3.87$. 


\section{Rubidiumtetrasulfid $\mathrm{Rb}_{2} \mathrm{~S}_{4} \cdot 2 \mathrm{H}_{2} \mathrm{O}$.}

Rubidium- und Cäsiumtetrasulfid werden nach der für Pentasulfide gültigen Arbeitsvorschrift bei Anwendung der berechneten Schwefelmengen erhalten.

Aus stark (auf ca. $6-8$ fach n.) eingeengten und geimpften Rubidiumtetrasulfidlösungen ${ }^{1}$ erhält man sehr kleine, kurze, prismatische Kristalle von lehmgelber Farbe, die durch Absaugen und Waschen mit Alkohol von Mutterlauge befreit werden können.

$0.2748 \mathrm{~g}$ Substanz: $0.7720 \mathrm{~g} \mathrm{BaSO}_{4}$.

$0.4912 \mathrm{~g} \quad, \quad: 0.3950 \mathrm{~g} \mathrm{Rb}_{2} \mathrm{SO}_{4}$.

Ber. S 38.25; Rb 51.00. — Gef. S 38.58; Rb 51.49.

Das Präparat ist zunächst ohne Schwefelabscheidung in Wasser löslich; von organischen Lösungsmitteln wird es nicht wesentlich verändert; beim Entwässern tritt Zersetzung ein.

Bei sehr langsamer Verdunstung von Rubidiumtetrasulfidlösungen im Exsikkator erhielten wir bisweilen ein äufserst labiles, in roten, gelbdurchscheinenden $\mathrm{Nadeln}$ kristallisierendes Produkt anderer $\mathrm{Zu}$ sammensetzung. Bei den Versuchen, die Kristalle durch Absaugen oder Aufpressen auf Ton von Mutterlauge zu befreien, zerfallen sie in ein feines Kristallpulver von den Eigenschaften des normalen Tetrasulfids, wobei es den Anschein hat, als ob gleichzeitig Wasser aufgenommen wird; denn bei dem Vorgange pflegt ein mehr oder weniger dünnflüssiger Brei von Nadeln und Mutterlauge zu einem ziemlich dichten, lehmgelben Kristallkuchen zu gestehen. Unter dem Mikroskope kann man diese Auflösung feuchter, in Kanadabalsam eingebetteter roter Nadeln in kleine gelbe Kriställchen gut verfolgen. Am meisten würde es für sich haben, in dem Präparate ein wasserärmeres Tetrasulfid zu erblicken, wenn nicht die Analyse eines einmal durch sehr vorsichtige Abtrennung von Mutterlauge isolierten Präparates für ein Sulfid von der Zusammensetzung $\mathrm{Rb}_{4} \mathrm{~S}_{9}$ spräche; indessen können wir die Existenz einer solchen,

${ }^{1}$ Konzentrierte Tetrasulfidlösungen sind in der Hitze dunkelrotgelb, bei Zimmertemperatur hellorange, in einer Kältemischung von fester Kohlensäure und Äther vor der Eisausscheidung schwach hellgelb. Da bei den festen Sulfiden (vergl. die Monosulfide) eine Hydratisierung ein Abblal'sen der Färbung zur Folge hat, so könnte man den Farbumschlag in Lösung als Kennzeichen einer mit wachsender Abkühlung fortschreitenden Zunahme der Wasserbindung ansehen; vergl. W. Britz, Zeitschr. phys. Chem. 40 (1902), 221. 
wenn auch labilen Verbindung nach dem vorliegenden Versuchsmaterial keineswegs als gesichert betrachten.

$$
\begin{array}{lll}
0.0836 \mathrm{~g} & \text { Substanz }: & 0.2706 \mathrm{~g} \mathrm{BaSO}_{4} . \\
0.0601 \mathrm{~g} \quad, \quad: & 0.1938 \mathrm{~g} \mathrm{BaSO}_{4} . \\
0.0986 \mathrm{~g} \quad, \quad: & 0.0817 \mathrm{~g} \mathrm{Rb}_{2} \mathrm{SO}_{4} .
\end{array}
$$

Gef. $\mathrm{S} 44.5,44.3 ; \mathrm{Rb} 53.1 ; \mathrm{Rb}: \mathrm{S}=2: 4.46$.

\section{Cäsiumtetrasulfid $\mathrm{Cs}_{2} \mathrm{~S}_{4}$.}

Cäsiumtetrasulfid kristallisiert leichter als Rubidiumtetrasulfid und zwar wasserfrei in rotgelben, nicht hygroskopischen, einige Zeit lang luftbeständigen Prismen, die ohne Schwefelabscheidung in Wasser löslich sind. Absoluter Alkohol vermag den Körper nicht zu lösen.

In verdünntem Alkohol ist das Präparat löslich und solche Lösungen sind an der Luft beständiger, als die wässerigen.

$$
\begin{array}{llll}
0.4483 \mathrm{~g} & \text { Substanz } & 1.0633 \mathrm{~g} \mathrm{BaSO}_{4} . \\
0.3715 \mathrm{~g} \quad " & : 0.8827 \mathrm{~g} \mathrm{BaSO}_{4} . \\
0.3371 \mathrm{~g} \quad " \quad: & 0.3090 \mathrm{~g} \mathrm{Cs}_{2} \mathrm{SO}_{4} .
\end{array}
$$

Ber. S 32.55; Cs 67.45. - Gef. S 32.57, 32.63; Cs 67.33.

Der früheren Schilderung der Darstellung von Pentasulfiden ist wenig neues hinzuzufügen. Bei Anwendung von 5-10 ccm stark konzentrierter (bis 5 fach normaler) Sulfidlösungen erhält man bei einiger Übung die Präparate analysenrein in einer Ausbeute von $75 \%\left(\mathrm{Rb}_{2} \mathrm{~S}_{5}\right)$ bis $85 \%\left(\mathrm{Cs}_{2} \mathrm{~S}_{5}\right)$. Aufser den beschriebenen wohlausgebildeten Kristallen bilden sich, wie bei der Rubidiumpentasulfidbereitung beobachtet wurde, bisweilen schon unmittelbar, nachdem das Einengen der Lösung beendet ist, schwarzglänzende Blättchen aus, die sich schollenartig an der Oberfläche ansammeln und als einseitig ausgebildete Individuen der roten Kristallgattung angesprochen werden können (O. WEIGEL).

II.

Eine einwandfreie Beantwortung der Grundfrage, in wie hohem Grade und in welchen Proportionen die Monosulfide der Alkalimetalle sich mit Schwefel verbinden können, steht, wie man sieht, auch in der Reihe des Rubidiums und Cäsiums noch aus. So sehr auch in der Reihe des Kaliums und Natriums durch die präpara- 
tive Chemie die Kenntnis einzelner Verbindungen dieser Art bereits gefördert ist, und so weitgehend auch durch die jüngsten Arbeiten die Lösungen von Schwefel in Alkalisulfid aufgeklärt sind, so bleibt einem.skeptischen Betrachter in der Charakterisierung der in festem Zustande erhaltenen Polysultide als wahre chemische Verbindungen noch manches zu wünschen übrig; bei rein präparativem Vorgehen hatte man nicht nur Di-, Tri-, Tetra- und Pentasulfide erhalten, sondern auch Sulfide von mittlerer Zusammensetzung, z. B. $\mathrm{K}_{4} \mathrm{~S}_{6} \cdot{ }^{1}$ Zieht man die Einheitlichkeit dieser Stoffe in Frage, so können natürlicherweise dabei auch Bedenken gegen die Einheitlichkeit jener entstehen.

Der von uns beschrittene Weg: Bearbeitung der Frage für die Alkalimetalle mit höchstem Atomgewicht, hatte zunächst gestattet, Pentasulfide zu bereiten, deren Charakterisierung innerhalb der üblichen Grenzen ziemlich lückenlos möglich war; die entsprechenden Tetrasulfide waren keineswegs mit der gleichen Schärfe definiert worden. Ausgehend von den nunmehr unschwer zugänglichen Pentasulfiden gelang es, wie im folgenden gezeigt wird, den Nachweis zu erbringen, dafs auch Tri-, Tetra- und Hexasulfide, aber keine thermostabilen Zwischenformen existieren. Dieses Resultat folgt aus einem auf Grund von Erstarrungskurven entworfenen Schmelzpunktsdiagramm. ${ }^{2}$

Die Aufnahme der Kurven, welche die Erstarrungspunkte der Sulfidschmelzen in Abhängigkeit von ihrem Schwefelgehalte darstellen, erfolgte in zwei Abschnitten, erstens zwischen Pentasulfid und reinem Schwefel, zweitens zwischen Disulfid und Pentasulfid.

Als Ausgangsmaterial für die ersten Serien konnten wir unmittelbar die auf nassem Wege gewonnenen Pentasulfide benutzen und die erforderlichen Mischungen durch Eintragen von Schwefel bereiten.

Zur Bereitung der für die zweiten Serien nötigen, wasserfreien Disulfide zeigte sich lediglich ein Weg gangbar: die Entziehung von Schwefel aus Pentasulfid bei höherer 'T'emperatur, am schnellsten im Wasserstoffstrome. Derartige Entschweflungen führten wir

1 Berzelus, Lebrbuch, 5. Aufl., II, 79, (1844), äufserte sich über die Existenz solcher Verbindungen unentschieden. - SchozNe, Dissert., Berlin 1867, Bull. Soc. des Nat. de Moscou 1866, IV, bestritt sie. - Bloxam, J. chem. soc. 67 (1895), 277; 77 (1900), 752, betrachtete sie wiederum als erwiesen.

\& Vergl. Tammane, Z. anorg. Chem. 37 (1903), 303; 45 (1905), 24; 47 (1905), 289. 
der besseren Regulierung halber in einem elektrisch geheizten Porzellanrohre unter pyrometrischer Temperaturmessung durch. Die Substanz befand sich im Innern des Rohres in Berliner Porzellantiegeln, die bei heller Rotglut von Disulfidschmelzen nicht erheblich angegriffen werden. Der Wasserstoff wurde elektrolytisch aus Natronlauge entwickelt.

Charakteristisch für ein gelungenes Entschwefeln ist ein beim Disulfid eintretender Farbwechsel. 2.5173 g Substanz verloren beim Anheizen bis $740^{\circ} 24.0 \%$ Schwefel, bei erneutem Anheizen auf $680^{\circ} 28.7 \%$, während sich für Disulfid ein Verlust von $29.0 \%$ Schwefel berechnet. Beide Schmelzen waren nach dem Erkalten gleichmärsig lebergelb und mit Sprüngen durchsetzt. Nach weiterem dreimaligen Anheizen bis $750^{\circ}$ und nachdem dann noch die Sub$\operatorname{stanz}$ eine halbe Stunde lang einer Temperatur von $810^{\circ}$ ausgesetzt gewesen war, war nur noch ein geringer weiterer Schwefelverlust . zu konstatieren. Das analytisch ermittelte Verhältnis $\mathrm{Rb}: \mathrm{S}=2: 1.92$ zeigt, dafs trotz der energischen Erhitzung eine wesentliche Reduktion über das Disulfid hinaus nicht stattgefunden hat. Man kann in diesem Befund, der durch spätere Beobachtungen bestätigt wurde, einen vorläufigen Beweis für die Existenz eines Disulfids, als eines Körpers von einem bestimmten Gebiete konstanter Schwefeltension erblicken. Gleichzeitig mit der Reduktion auf Disulfid wurde die Masse dunkelrot, bisweilen mit blaurotem Oberflächenschimmer; dünne Splitter waren rotdurchscheinend. Übergielst man ein solches Disulfid mit Wasser, so hat man den ungewöhnlichen Anblick, wie sich eine intensiv gefärbte Substanz farblos löst. Allerdings ist diese Lösung stets mit einer Spur aus dem Porzellan herrührenden Kisensulfids verunreinigt, und man könnte daher Bedenken tragen, jene rote Farbe als Eigenfarbe des Disulfids anzusprechen. Indessen konnte ein Zusammenhang zwischen der Farbtiefe und der Menge des Eisensulfids weder erkannt werden, wenn man als Gefäfsmaterial gewöhnliches, stärker eisenhaltiges Porzellan anwandte, noch wenn man die Eisensulfidmenge durch Zusatz von Eisen zu der fertigen Disulfidschmelze stark steigerte. ${ }^{\beth}$ Wie scharf im übrigen die Farbe

1 Einen bündigen Beweis füx die Farbe des wasserfreien Disulfids können wir leider nicht beibringen, da ein geeignetes, völlig eisenfreies Gefälsmaterial nicht zur Verfügung steht. Quarz wird durch geschmolzenes Alkalidisulfid völlig entglast. Mit Bestimmtheit kann jedoch behauptet werden, dals die Lösungen von Disulfid nicht rotgelb sind, wie solche bei den S. 301-302 mitgeteilten Versuchen erbalten worden waren. 
eines Disulfids von einer Trisulfidbeimengung beeinflufst wird, zeigte die Analyse eines rein gelben Entschweflungsproduktes, in dem sich Rubidium zu Schwefel verhielt wie $2: 2.038$.

Die Entschweflungen von Cäsiumpentasulfid erfordern, wie es den Anschein hat, höhere Temperaturen. Nach $1-1^{1} / 2$ stündigem Erhitzen von ca. $11 \mathrm{~g}$ Pentasulfid auf $810^{\circ}$ war die Reduktion beendet.

$0.1673 \mathrm{~g}$ Substanz: $0.2367 \mathrm{~g} \mathrm{BaSO}_{4}$.

$0.1501 \mathrm{~g} \quad, \quad: 0.2120 \mathrm{~g} \mathrm{BaSO}_{4}$.

$0.2631 \mathrm{~g} \quad, \quad: 0.2875 \mathrm{~g} \mathrm{Cs}_{2} \mathrm{SO}_{4}$.

$0.1152 \mathrm{~g} \quad, \quad: 0.1258 \mathrm{~g} \mathrm{Cs}_{2} \mathrm{SO}_{4}$.

Ber. für $\mathrm{Cs}_{2} \mathrm{~S}_{2}$, S 19.44; Cs 80.56. - Gef. S 19.43, 19.40; Cs $80.27,80.21$.

Nach Abschlufs einer Cäsiumpentasulfidentschweflung findet sich auf der Innenseite des Porzellanrohres, an dem Pyrometerschutzrohre und dem Ableitungsrohr, ein hellziegelroter Sulfidbeschlag. Wie bei den Chloriden nimmt also die Flüchtigkeit der Sulfide mit wachsendem Molekulargewicht zu.

In der Rubidiumreibe wurden, die Vorversuche ungerechnet, drei Serien von Erstarrungsmessungen ausgeführt, die vom Rubidiumdisulfid ausgingen, ferner eine Serie zwischen Pentasulfid und reinem Schwefel und schliefslich eine solche zwischen Tetrasulfid und Pentasulfid. Für die Cäsiumreihe wurden zwischen Pentasulfid und Schwefel zwei vollständige Serien ausgearbeitet, zwischen Disulfid und Pentasulfid eine vollständige und schliefslich eine solche zwischen Tetrasulfid und Pentasulfid. Eine Kontrolle für die Rubidiumpentasulfidserie der Erstarrungs punkte bildete die Ermittelung der Schmelzpunkte von Gemischen in dem gleichen Gebiete'.

Zur Ausführung der Versuche ${ }^{1}$ wurde das analysierte Ausgangsmaterial in ein kleines aus Duraxglas gefertigtes Schmelzgefäls gebracht, das einen seitlichen Stutzen zur Einführung eines indifferenten Gasstromes besals und durch einen Stopfen mit Durchbohrungen zur Aufnahme eines geeichten Quecksilberthermometers, eines kleinen Glasrührers und eines Impfstiftes verschlossen war. Zum Anheizen benutzten wir im Anfang Paraffinbäder und ebensolche zum Konstanthalten der Aufsentemperatur während der Erstarrung. Bequemer erwies sich bei den zeitlich zuletzt ausgeführten Versuchen

1 Über Einzelheiten, vergl. E. WiLke-Dörrurt, Über Sulfide und Polysulfide des Rubidiums und Cäsiums. Dissert., Göttingen 1906. 
als Heizquelle ein einfacher Kryptolofen. Bei Versuchen in der Gegend der Disulfide mufste mit Porzellanapparaten und pyrometrischer Temperaturmessung gearbeitet werden. Als Schmelzgefäls diente dann ein in einem starkwandigen Jenenser Reagensglase stehender Porzellantiegel; Pyrometerschutzrohr und Impfstift, beide in möglichst kleinen Dimensionen, waren ebenfalls aus Porzellan. Zum Rühren diente das Pyrometerschutzrohr. Das Pyrometer war durch Bestimmung der Erstarrungspunkte von Zink, Blei und Zinn geeicht und aufserdem mit dem Quecksilberthermometer verglichen. Die folgenden Temperaturangaben des Quecksilberthermometers sind unter Berücksichtigung der mittleren Fadentemperaturen korrigiert. Die Herstellung der Mischungen von Sulfid mit Schwefel erfolgte, ähnlich wie bei Molekulargewichtsbestimmungen, durch Eintragen von in plastischem Zustande passend zugeschnittenen Stücken Schwefels im Schmelzgefälse selbst. ${ }^{1}$

Die Temperaturablesung erfolgte alle 10 Sekunden. Die Genauigkeit der Ablesung betrug für das Quecksilberthermometer ein Zehntel, für das Pyrometer einen vollen Grad.

Die Schwierigkeiten der Versuche lagen darin, dafs die Schmelzen sehr leicht oxydierbar und zum Teil hygroskopisch sind; ferner dafs der Temperaturausgleich innerhalb der Schmelzen, z. B. zwischen Cäsiumdisulfid und Trisulfid, zu wünschen übrig läfst, schliefslich, dals wir besonders im Anfang der Arbeit genötigt waren, mit sehr kleinen Substanzmengen, etwa $3 \mathrm{~g}$, zu arbeiten. Die Hauptschwierigkeit trat bei den niederen Polysulfiden zutage, deren Gemische zum Teil mit sehr geringer Kristallisationsgeschwindigkeit, zum Teil völlig amorph erstarren.

Eindringen von Wasser und Luft während der Versuche wurde dadurch vermieden, dafs reiner, getrockneter Wasserstoff oder luftfreier, trockener Stickstoff durch den erwähnten seitlichen Ansatz in den Schmelzapparat ein- und durch die Öffnungen des Stopfens ausgeleitet wurde. Bei Temperaturen über $240^{\circ} \mathrm{kam}$ nur Stickstoff in Frage. Zur Beförderung des Temperaturausgleiches, sowie zur Vermeidung von Unterkühlung werden die erstarrenden Schmelzen so weit als irgend tunlich durchrührt. Unbedingt erforderlich ist es, zur richtigen, jedesmal erst auszuprobierenden Zeit zu impfen.

1 Die Vereinigung von Schmelze und Schwefel ging aufser bei sehr schwefelarmen Schmelzen glatt von statten; bei diesen tritt die Reaktion unter leichtem Zischen und bisweilen mit geringfügiger Schwefelverdampfung ein. Über die Korrektur der eingetragenen Schwefelmenge vergl. unten. 
Der Impfstift hing zwischen den einzelnen Versuchen so, dals die Impfmasse sich im oberen, mit indifferentem Gas gefüllten Teil des Apparates befand. Wenn auch somit für jeden häufig geprüften Wert der Erstarrungs- und eutektischen Punkte weitgehend Gewähr geleistet werden kann, so war es doch schon wegen der von Versuch zu Versuch durch Eintragen von Schwefel veränderten Masse der Schmelze nicht möglich, die eutektischen Zeiten in einer von Willkür völlig freien. Weise zu bestimmen. Ebensowenig erwies sich eine Kontrolle der thermischen Ergebnisse durch Untersuchung von Schliffstücken ausführbar.

Die Genauigkeit der einzelnen Bestimmungen ist natürlich sehr verschieden und hängt in erster Linie von der Neigung der Schmelzkurve ab. Während z. B. in der Gegend von Pentasulfid viele Werte bis auf $1^{0}$, und weniger, genau sind, können nahe dem Disulfid, der steilsten Stelle der Kurve, leicht Fehler von 10 und mehr Grad vorgekommen sein.

Bei der Kritik der Versuche war den einzelnen Punkten ganz verschiedene Wertschätzung beizulegen. So mufsten Punkte, die an den Grenzen amorpher Gebiete nur ungenau bestimmt werden konnten, zugunsten vorhergehender oder nachfolgender verworfen werden. Ferner bedurfte der Prozentgehalt der Schmelzen an Schwefel einer Korrektur, weil es bei oft wochenlanger Dauer einer Serie nicht möglich war, jeden Schwefelverlust durch Verdampfen auszuschliefsen und unter Umständen auch an den oberen Teilen des Rührers, Thermometers oder Impfstiftes haftende Substanz der Durchmischung mit neu eingetragenen Schwefelportionen entzogen blieb. Zur Korrektur der aus der Analyse des Ausgangsmaterials und dem Gewichte des eingetragenen Schwefels berechneten Prozente Schwefel diente die Analyse des Endproduktes der betreffenden Serie. Ein Plus oder Minus an Schwefel wurde dann proportional auf die einzelnen Schwefelportionen verteilt. Auf diese Weise konnte der gegenseitige Anschlufs der verschiedenen Serien befriedigend erreicht werden. Zur Deutung der gefundenen Knickund Haltepunkte auf den Erstarrungskurven waren Beobachtungen über die Konsistenz der Schmelze, die beim Rühren leicht ermittelt werden konnte, von grofsem Werte, besonders, wenn durch undeutliche oder mangelnde Ausbildung einer der zwei im allgemeinen zu erwartenden Punkte Zweifel entstanden, ob der beobachtete ein Frstarrungs- oder ein eutektischer Punkt war. Von der Verwertung für die definitive tabellarische Zusammenstellung wurden diejenigen 
Tabelle 1.

Rubidiumpolysulfide.

\begin{tabular}{|c|c|c|c|c|}
\hline $\mathrm{Nr}$. & $\begin{array}{c}\% \\
\text { Schwefel } \\
\end{array}$ & $\begin{array}{c}\text { Erstarrung } \\
\text { in }{ }^{0} \\
\end{array}$ & $\begin{array}{l}\text { Eutekt. Er- } \\
\text { starrung in }{ }^{\circ}\end{array}$ & Bemerkungen \\
\hline 1 & 27.45 & etwa 420 & - & \multirow{12}{*}{ Unterkühlung bis $158^{\circ}$} \\
\hline 2 & 30.21 & $\Rightarrow \quad 380$ & 一 & \\
\hline 3 & 32.61 & 231.5 & - & \\
\hline 4 & 32.84 & - & - & \\
\hline 5 & 32.85 & - & 199.5 & \\
\hline 6 & 33.03 & 227 & - & \\
\hline 7 & 33.47 & 221.5 & - & \\
\hline 8 & 33.69 & - & 202 & \\
\hline 9 & 33.90 & 216 & - & \\
\hline 10 & 34.28 & - & 200 & \\
\hline 11 & 34.46 & 211.5 & - & \\
\hline 12 & 34.73 & - & 199 & \\
\hline 13 & 34.84 & 210.5 & - & \multirow{19}{*}{ Mittel: $200^{\circ}$} \\
\hline 14 & 35.03 & 214 & - & \\
\hline 15 & 35.38 & 一 & 198 & \\
\hline 16 & 35.48 & 212 & - & \\
\hline 17 & 35.60 & - & 200 & \\
\hline 18 & 35.95 & 211.5 & 一 & \\
\hline 19 & 35.96 & - & 200 & \\
\hline 20 & 35.99 & - & 200 & \\
\hline 21 & 36.22 & - & 199 & \\
\hline 22 & 36.42 & - & 200.5 & \\
\hline 23 & 36.57 & 195 & - & \\
\hline 24 & 36.97 & 195 & - & \\
\hline 25 & 37.33 & 190 & - & \\
\hline 26 & 37.85 & 185.5 & {$[154]$} & \\
\hline 27 & 38.40 & - & {$[155]$} & \\
\hline 28 & 38.91 & - & 147.5 & \\
\hline 29 & 39.40 & - & 148.5 & \\
\hline 30 & 39.94 & - & 148.5 & \\
\hline 31 & 40.05 & 164.5 & - & \\
\hline 32 & 40.47 & - & 148.5 & \multirow[t]{8}{*}{ Mittel: $148.5^{\circ}$} \\
\hline 33 & 40.97 & - & 150 & \\
\hline 34 & 41.44 & {$[157]$} & - & \\
\hline 35 & 41.47 & - & 148 & \\
\hline 36 & 41.95 & - & 148.5 & \\
\hline 37 & 42.13 & 153 & - & \\
\hline 38 & 42.62 & 157.5 & - & \\
\hline 39 & 43.14 & 160.4 & - & \\
\hline 40 & 43.46 & 171.5 & 159.3 & \multirow{2}{*}{ Mittel: $159.5^{\circ}$} \\
\hline 41 & 43.78 & 185.5 & - & \\
\hline
\end{tabular}


Tabelle 1 (Fortsetzung).

\begin{tabular}{|c|c|c|c|c|}
\hline Nr. & $\begin{array}{c}\% \\
\text { Schwefel }\end{array}$ & $\begin{array}{l}\text { Erstarrung } \\
\quad \text { in }{ }^{0}\end{array}$ & $\begin{array}{l}\text { Eutekt. Er- } \\
\text { starrung in }\end{array}$ & Bemerkungen \\
\hline 42 & 44.03 & 185 & 159 & \multirow{21}{*}{ Mittel: $159.5^{0}$} \\
\hline 43 & 44.09 & 195.5 & 160 & \\
\hline 44 & 44.42 & 199 & 158 & \\
\hline 45 & 44.74 & 204.5 & 159 & \\
\hline 46 & 45.04 & 211.5 & 159 & \\
\hline 47 & 45.12 & 212 & - & \\
\hline 48 & 45.36 & 215 & - & \\
\hline 49 & 45.44 & 217.3 & - & \\
\hline 50 & 45.67 & 222 & - & \\
\hline 51 & 45.78 & 222.4 & $\cdots$ & \\
\hline 52 & 46.12 & 227 & - & \\
\hline 53 & 46.52 & 229 & - & \\
\hline 54 & 46.88 & 230 & $\cdots$ & \\
\hline $5 \overline{5}$ & 47.24 & 230 & - & \\
\hline 56 & 47.74 & 231 & - & \\
\hline 57 & 48.21 & 230 & - & \\
\hline 58 & 48.66 & 228 & 190 & \\
\hline 59 & 49.11 & 228 & 190 & \\
\hline 60 & 49.22 & 224.5 & 189.8 & \\
\hline 61 & 50.51 & 221.0 & 189.8 & \\
\hline 62 & 51.51 & 215.0 & 189.8 & \\
\hline 63 & 51.98 & 212.5 & 189.8 & \multirow[t]{8}{*}{ Mittel: $189.8^{\circ}$} \\
\hline 64 & 52.59 & 207.8 & 190.0 & \\
\hline 65 & 53.05 & 206.0 & 190.0 & \\
\hline 66 & 53.30 & 200 & 189.8 & \\
\hline $6 \vec{i}$ & 53.54 & 200 & 189.8 & \\
\hline 68 & 53.77 & 196 & 189.4 & \\
\hline 69 & 53.97 & 188 & - & \\
\hline 70 & 54.43 & 185.5 & - & \\
\hline 71 & 55.29 & 185.5 & - & \multirow{4}{*}{ Mittel: $184.6^{\circ}$} \\
\hline 72 & 56.09 & 184.5 & - & \\
\hline $73 a$ & - & 183.0 & - & \\
\hline $73 \mathrm{~b}$ & - & 117.5 & - & \\
\hline
\end{tabular}

Resultate ausgeschlossen, die entweder in Vorversuchen unter noch wenig geeigneten Abkühlungs- und Impfbedingungen gefunden worden waren, ferner ein Teil solcher Serien, die durch Differenzen im Prozentgehalt einen gegenseitigen, glatten Anschlufs zu dem vollständigen Diagramm nicht gestatteten, selbst wenn sie in sich $\mathrm{zu}$ einer Bestätigung einzelner Teile der Kurve wohl geeignet sind, und schliefslich in ganz seltenen Fällen solche Punkte, die, durch grobe Febler entstellt, aus einer sonst gut bestätigten Strecke herausfallen. 
Tabelle 2.

Cäsiumpolysulfide.

\begin{tabular}{|c|c|c|c|c|}
\hline $\mathrm{Nr}$. & $\begin{array}{c}0 \\
\text { Schwefel }\end{array}$ & $\begin{array}{l}\text { Erstarrung } \\
\quad \text { in }{ }^{0}\end{array}$ & $\begin{array}{l}\text { Eutekt. Er- } \\
\text { starrung in }{ }^{\circ}\end{array}$ & Bemerkungen \\
\hline 1 & 19.43 & etwa 460 & - & \multirow{17}{*}{ Mittel: $205.5^{\circ}$} \\
\hline 2 & 19.61 & 410 & - & \\
\hline 3 & 20.43 & 398 & - & \\
\hline 4 & 21.93 & 341 & - & \\
\hline$\tilde{5}$ & 22.84 & - & 206 & \\
\hline 6 & 23.08 & - & 206 & \\
\hline 7 & 24.43 & - & 206 & \\
\hline 8 & 24.67 & - & 204 & \\
\hline 9 & 25.25 & {$[192.5]$} & $m$ & \\
\hline 10 & 25.68 & amorph & amorph & \\
\hline 11 & 26.16 & $"$ & $"$ & \\
\hline 12 & 26.76 & , & $"$ & \\
\hline 13 & 26.97 & $n$ & $"$ & \\
\hline 14 & 27.46 & $n$ & $"$ & \\
\hline 15 & 27.73 & - & {$[143]$} & \\
\hline 16 & 28.08 & - & 150 & \\
\hline 17 & 28.61 & - & 151 & \\
\hline 18 & 29.57 & - & 150 & \multirow{7}{*}{ Mittel: $151^{\circ}$} \\
\hline 19 & 30.56 & 153 & - & \\
\hline 20 & 31.04 & 157.5 & 151 & \\
\hline 21 & 31.47 & - & 152.5 & \\
\hline 22 & 32.14 & 158.5 & - & \\
\hline 23 & 32.75 & 160 & - & \\
\hline 24 & 33.47 & 185 & 160.5 & \\
\hline 25 & 34.22 & 196 & 158.5 & \multirow{5}{*}{ Mittel : $159.5^{\circ}$} \\
\hline 26 & 34.88 & 204 & 159.5 & \\
\hline 27 & 35.63 & 209 & - & \\
\hline 28 & 36.18 & 209 & - & \\
\hline 29 & 39.04 & 198.8 & - & \\
\hline 30 & 39.18 & 197.9 & 179.3 & \multirow{11}{*}{ Mittel: $178^{\circ}$} \\
\hline 31 & 39.66 & 194.8 & 177.0 & \\
\hline 32 & 40.15 & 189.0 & - & \\
\hline 33 & 41.31 & 184.5 & - & \\
\hline 34 & 41.87 & 184.5 & - & \\
\hline 35 & 42.44 & 185.8 & - & \\
\hline 36 & 42.68 & 183.2 & - & \\
\hline 37 & 42.89 & 179 & - & \\
\hline 38 & 43.09 & 178 & - & \\
\hline 39 & 43.30 & 175 & - & \\
\hline 40 & 44.17 & 172.8 & - & \\
\hline 41 & 44.79 & 172.8 & - & \multirow[t]{2}{*}{ Mittel: $172.8^{\circ}$} \\
\hline 42 & 45.60 & 172.8 & - & \\
\hline
\end{tabular}




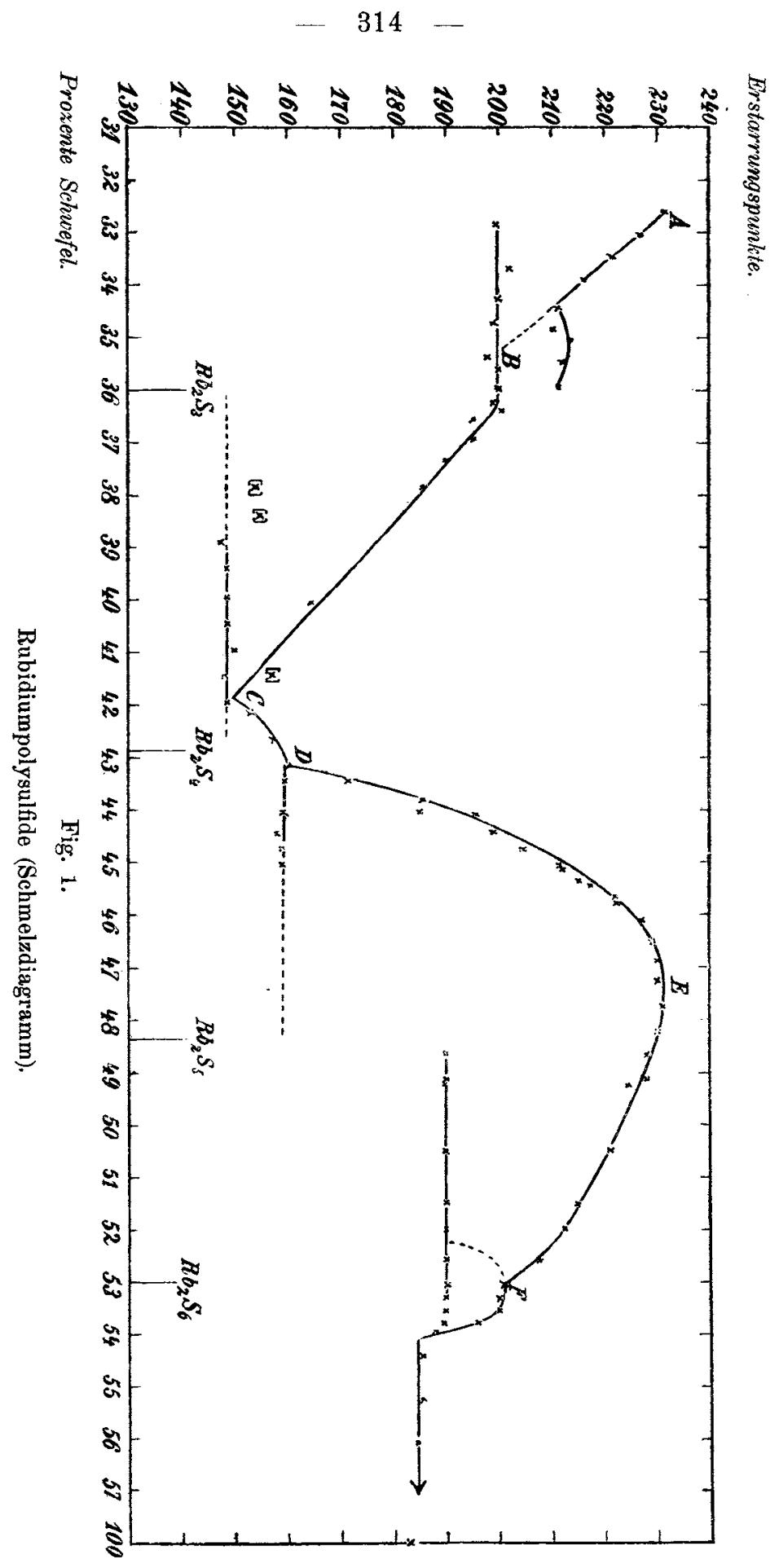


Aus der Kurventafel Fig. 1 ergeben sich für die Rubidiumpolysulfide vier eutektische Horizontalen: eine solche zwischen Di- und Trisulfid bei $200^{\circ}$, eine zweite zwischen Tri- und Tetrasulfid bei $148.5^{\circ}$, eine dritte zwischen Tetra- und Pentasulfid bei $159.5^{\circ}$ und eine letzte zwischen Penta- und Hexasulfid bei $189.8^{\circ}$. Ein ausgeprägtes Maximum beobachtet man in $E$ für das Pentasulfid, das demnach thermisch der beständigste der vorliegenden Körper ist. Für die Existenz des Tetrasulfids spricht aufser dem Wechsel der eutektischen Horizontalen zwischen 42 und $43 \%$ die mit dem Pentasulfidast einen deutlichen Winkel bildende Kurve $C D$. Die Ausbildung eines Maximums ist dadurch verhindert, dafs der Stoff unterhalb seines Schmelzpunktes zerfällt. Ein ebensolches verdecktes Maximum findet man für Trisulfid und Hexasulfid. Die Bestimmungen in der Gegend des Trisulfids erforderten besonders zahlreiche Belege, weil sich hier die erwähnte Kristallisationsträgheit geltend macht und zwischen Di- und Trisulfid vollkommen amorphes Erstarren eintritt. Beim Trisulfid beobachtet man, je nachdem man die Impfbedingungen glücklich getroffen hat, Erstarrungspunkte, die für ein und dieselbe Schmelze über $10^{\circ}$ auseinanderliegen. Man könnte vielleicht denken, dafs in dem einen Falle Punkte eines Trisulfidmaximums, in dem anderen solche eines verdeckten Maximums zur Beobachtung gelangen. Zwischen Trisulfid und Disulfid steigt der Erstarrungspunkt um etwa $200^{\circ}$. Es liefsen sich nur die ersten und die letzten Punkte (diese pyrometrisch) festlegen. ${ }^{1}$ Wir können daher nicht mit Bestimmtheit behaupten, $o b$ zwischen diesen beiden Verbindungen noch eine dritte existiert, oder ob etwa $z$ wischen den zugehörigen Temperaturen eine polymorphe Umwandlung des Disulfids stattfindet. Für einen ausgezeichneten Punkt in diesem Gebiete spricht die schwache Neigung des untersten Stückes $A B$ der Verbindungskurve, die notwendigerweise, um den Anschlufs an die hohen Werte zu erreichen, bei einem mittleren Prozentgehalte durch einen Knick unterbrochen sein mufs. Eines der mittleren Gemische mit $32.84 \%$ Schwefel konnte erst nach enormer Unterkühlung bei $158^{\circ}$ zur Kristallisation gebracht werden. Rubidiumbexasulfid ist durch ein verdecktes Maximum zwischen 53 und 54\% Schwefel charakterisiert. Unmittelbar hinter dem Hexasulfid, bei $54.1 \%$, hört die Mischbarkeit der Sulfidschmelzen mit Schwefel auf. Man erhält eine fünfte Horizontale bei $185^{\circ}$, die den Erstarrungspunkt eines mit Schwefel ge-

1 /wischen diesen liegt ein Gebiet amorpher Erstarrung. 


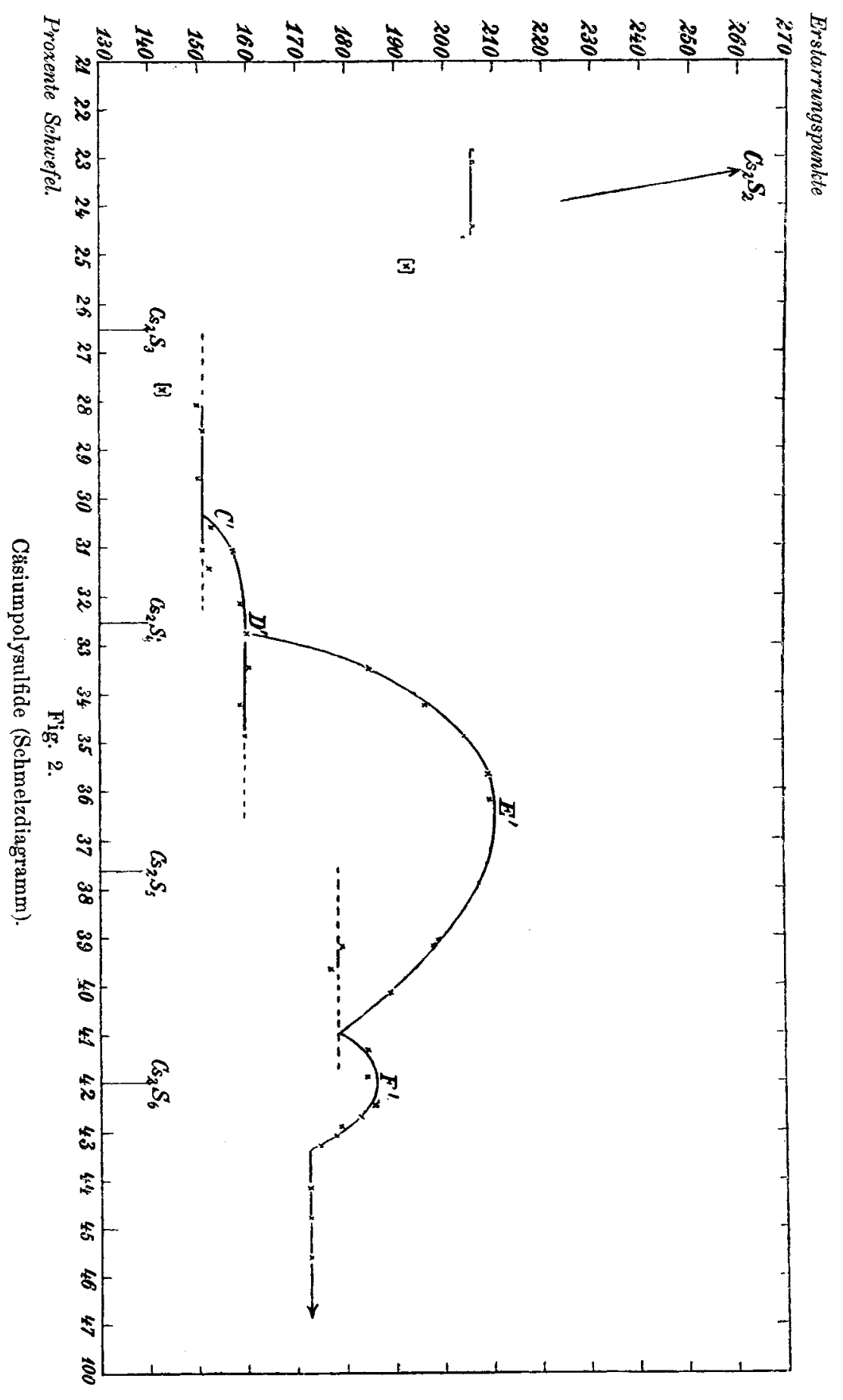


sättigten Hexasulfids angibt. Es wurde dies aufs beste bestätigt, als mit etwa $4 \mathrm{~g}$, d. h. der gleichen Menge Schwefel, wie bereits Substanz vorhanden war, durchmischt und sowohl für den oberen Teil der Schmelze, wie für den unteren die Erstarrungskurve aufgenommen wurde. Für den unteren Teil war der Wert innerhalb eines Prozentes der gleiche geblieben, die Schmelze war also bereits vorher gesättigt. Andererseits löst auch geschmolzener Schwefel kein Polysulfid auf, wie die rein gelbe Farbe der nach dem Erstarren scharf abgegrenzten oberen Schicht und noch schlagender ihr Erstarrungspunkt $117.5^{\circ}$ bewies.

In der Cäsiumpolysulfidkurve ist bemerkenswert die bessere Ausbildung des Hexasulfidmaximums und die Ausdehnung der amorphen Gebiete bei Trisulfid, so dafs man zu dessen Identifizierung lediglich auf den Wechsel der eutektischen Horizontalen $205.5^{\circ}$ und $151^{\circ}$ angewiesen ist. Die Richtung, in der die Kurve möglicherweise zwischen Di- und Trisulfid verläuft, läfst sich aus den pyrometrisch ermittelten Werten über $300^{\circ}$ ableiten. In der Kurventafel ist sie durch einen Pfeil bezeichnet. Die Sättigung von Cäsiumbexasulfid mit Schwefel ist bei $43.3 \%$ Schwefel erreicht; darüber hinaus wird, wie ein besonderer Versuch bewies, auch bei sehr grofsem Schwefelüberschufs weder Schwefel aufgenommen, noch ist dieser imstande, Hexasulfid zu lösen. Ebensowenig, wie beim Rubidium findet sich irgendwo eine Andeutung, dafs aufser den genannten noch andere Polysulfide stabiler Existenz vorhanden sind.

Einen Vergleich zwischen den Rubidium- und Cäsiumpolysulfiden gestattet die Tabelle 3, aus der hervorgeht, dafs die Schmelzpunkte in beiden Reihen in inversem Sinne von der Zusammensetzung

Tabelle 3.

Schmelzpunktsvergleich.

\begin{tabular}{|c|c|c|c|}
\hline Verbindungen u. Eutektika & Rubidium & Cäsium & Differenz \\
\hline Disulfid & etwa $420^{\circ}$ & etwa $460^{\circ}$ & $+40^{\circ}$ \\
\hline Eutektikum $_{2,3}$ & 200 & 205.5 & +5.5 \\
\hline Trisulfid & 213 & \multicolumn{2}{|c|}{217 (interpoliert) $[+4]$} \\
\hline Eutektikum $_{3}, 4$ & 148.5 & 151 & +2.5 \\
\hline Tetrasulfid & $>160$ & $>160$ & - \\
\hline Eutektikum $_{4,5}$ & 159.5 & 159.5 & \pm 0 \\
\hline Pentasulfid & 231 & 210 & -21 \\
\hline Eutektikum $_{5,6}$ & 189.8 & 178 & -11.8 \\
\hline Hexasulfid & 201 & 186 & -15 \\
\hline Gesättigte Lösung & 184.6 & 172.8 & -11.8 \\
\hline
\end{tabular}


abhängen, je nachdem es sich um höher oder niedriger molekulare Verbindungen handelt, ein Ergebnis, das auch für manche andere physikalische Daten, z. B. die Löslichkeit der Alkalimetallverbindungen gilt.

Die Farbe der Polysulfidschmelzen ist in beiden Fällen die gleiche: Die Disulfide sind rot; von Trisulfid bis zur gesättigten Hexasulfidlösung vertieft sich die Farbe von gelb über rot bis auf braunrot.

Das Gesamtergebnis stellt sich für unseren Fall dar als eine vollkommene Beweisführung für die seit altersher angenommene Zusammensetzung der Alkalimetallpolysulfide mit der Ergänzung, dafs die Absättigung mit Schwefel erst beim Hexasulfid erreicht ist, und dals thermostabile Schwefelverbindungen von der Formel $\mathrm{M}_{2} \mathrm{~S}_{n}$, worin $n$ eine gebrochene Zahl bedeutet, nicht existieren. ${ }^{3}$ Der erste Punkt korrespondiert mit den Küsterschen Beobachtungen über die Löslichkeit von Schwefel in Natronlauge, - Küster fand bei gewissen Konzentrationen den Wert von 5 Atomen überschritten steht dagegen nicht in Übereinstimmung mit dem. Verhalten des Kaliumpentasulfids, das, wie BERzEurus fand, durch Zusammenschmelzen mit Schwefel nicht höher sulfuriert werden konnte.

Die vorliegende Arbeit wurde teils im chemischen Institute der Universität Göttingen, teils im chemischen Laboratorium der Bergakademie Clausthal ausgeführt.

Bei den letzten Versuchen unterstützte uns Herr F. KAUFMANN, wofür wir ihm unsern besten Dank aussprechen möchten.

1 Die Existenzmöglichkeit solcher Verbindungen unter anderen Bedingungen kann natürlich nicht in Abrede gestellt werden, ebensowenig, wie die von Hepta- und Enneasulfiden, für welche besonders Beispiele aus der Ammoniumreihe vorliegen.

Clausthal, Chem. Laboratorium der Bergakademie.

Bei der Redaktion eingegangen am 9. Januar 1906. 